\title{
Risk factors for calcineurin inhibitor nephrotoxicity after renal transplantation: a systematic review and meta-analysis
}

This article was published in the following Dove Press journal: Drug Design, Development and Therapy

\author{
Tianyi Xia \\ Sang Zhu \\ Yan Wen \\ Shouhong Gao \\ Mingming $\mathrm{Li}$ \\ Xia Tao \\ Feng Zhang \\ Wansheng Chen \\ Department of Pharmacy, \\ Changzheng Hospital, Second \\ Military Medical University, Shanghai, \\ People's Republic of China
}

Correspondence: Wansheng Chen; Feng Zhang

Department of Pharmacy, Changzheng Hospital, Second Military Medical University, Fengyang Road No 415, Shanghai 200003, People's

Republic of China

Email chenwansheng@smmu.edu.cn; zhangfeng@smmu.edu.cn
Background: Nephrotoxicity of calcineurin inhibitors (CNIs) is the major concern for long-term allograft survival despite its predominant role in current immunosuppressive regime after renal transplantation. CNI nephrotoxicity is multifactorial with demographic, environmental, and pharmacogenetic flexibility, whereas studies indicating risk factors for CNI nephrotoxicity obtained incomplete or conflicting results.

Methods: A systematic review and meta-analysis of risk factors for CNI nephrotoxicity was performed on all retrieved studies through a comprehensive research of network database. Data were analyzed by Review Manager 5.2 with heterogeneity assessed using the Cochrane Q and $I^{2}$ tests. CNI nephrotoxicity was primarily indicated with protocol biopsy or index-based clinical diagnosis, and the secondary outcome was defined as delayed graft function.

Results: Twelve observational studies containing a total of 2,849 cases were identified. Donor age (odds ratio [OR], 1.01; 95\% CI, 1.01-1.03; $p=0.02)$, recipient zero-time arteriosclerosis $(\mathrm{OR}$, 1.44; 95\% CI, 1.04-1.99; $p=0.03$ ), and $C Y P 3 A 5 * 3 / * 3$ genotype (OR, 2.80; 95\% CI, 2.63-2.98; $p=0.00$ ) were confirmed as risk factors for CNI nephrotoxicity. Subgroup and sensitivity analysis claimed donor age as a significant contributor in Asian and Caucasian areas.

Conclusion: Older donor age, recipient zero-time arteriosclerosis, and CYP $3 A 5^{*} 3 / * 3$ genotype might add up the risk for CNI nephrotoxicity, which could be interpreted into a robust biomarker system.

Keywords: calcineurin inhibitor, transplantation, nephrotoxicity, risk factor, systematic review, meta-analysis

\section{Introduction}

Organ transplantation across alloantigen barriers is widely applicable with reduced acute rejection (AR) rate and excellent 1-year graft survival, mainly because of the ongoing efforts toward improving immunosuppressive regimes. ${ }^{1,2}$ Calcineurin inhibitors (CNIs), such as tacrolimus (FK506) and cyclosporine A (CsA), gain universal application as influential immunosuppressants since 1980s, while the nephrotoxicity concomitant with its therapeutic effects has been incriminated in the lack of improvement in longterm graft survival, even in grafts with excellent function. Nephrotoxicity secondary to CNIs is reported to occur in $76 \%-94 \%$ of the renal transplant recipients. ${ }^{3}$

CNI nephrotoxicity clinically presented as the abrupt increase in serum creatinine (Scr) disturbed homeostasis and nodular arteriolar hyalinosis (as histologic hallmark) without morphologic changes, resulting in reversible acute/irreversible chronic allograft lesions. ${ }^{4-6}$ Surveillance of posttransplantation CNI nephrotoxicity is of paramount significance for timely diagnosis. Available diagnostic methods 
including clinical presentation, biochemical parameters, and renal biopsy always failed preemptive intervention due to large heterogeneity and interrater variability with focus on medullar damage instead of the tubules. In recent decades, metabolite phenotyping offered a better mechanistic understanding of CNI-induced tubule damage through the modern technology "omics," an unbiased, holistic, and "system biology" approach for progressive clinical utilization, identifying several innovative biomarkers (KIM-1, TIMP-2, IGFBP-7, etc.) with better sensitivity and selectivity over creatinine and estimated glomerular filtration (eGFR). ${ }^{7-9}$ Nevertheless, none of the biomarkers have made their way into today's clinical practice as a universally accepted diagnostic tool because it is difficult to identify the exact pathophysiological alterations in the wealth of background matrix information. Hence, discovery of the undermining biomarkers for CNI nephrotoxicity will be a long march.

As noted earlier, CNI nephrotoxicity is patient, medication, and environment specific, ${ }^{10}$ for which a rational combination may guide the mapping of the molecular landscape of the disease entities and the laws of metabolite alterations. In light of this, theories about the mechanisms leading to CNI nephrotoxicity focus on pharmacokinetics (PK)/pharmacodynamics (PD) and pharmacogenetics of CNI metabolism, in addition to the above-mentioned pathological and molecular mechanisms. Moreover, there is convincing evidence that long-term renal dysfunction can be influenced by both immune and nonimmune mechanisms against a background of various donor and recipient factors. ${ }^{11}$ In this regard, CNI nephrotoxicity is recognized to be subject to immune factors such as histocompatibility, AR, and subclinical acute rejection (SAR), as well as other nonimmune factors including donor age, gender, and cold/warm ischemia time, and then CNI nephrotoxicity accelerates allograft decay. ${ }^{12,13}$ Accordingly, a combination of several clinical, biological, and genetic predictive parameters with further interpretation of influential "omics-based" biomarkers may provide cogent clues to pick up early physiopathologic alterations of CNI nephrotoxicity, thereby bringing first-time intervention for "at-risk" patients. ${ }^{14,15}$ However, data from these investigations are controversial probably due to study design and subject heterogeneity.

Altogether, the multifaceted puzzle of CNI nephrotoxicity is a chronically progressive, largely identifiable, and potentially amendable procedure, and thus, identification of risk factors for CNI nephrotoxicity can be of clinical impact for renal failure management in posttransplantation. Herein, we initiatively conducted a systematic review, which focused on the potential risk factors for CNI nephrotoxicity in the actual context of renal transplantation to make sure their feasibility in clinical practice as biomarker candidates. In addition, meta-analyses were performed to resolve the discrepancies among the investigated explorations using data filtration for the persuasive and impactful ones. The proposed study would not only shed light on the cogent risk factors but also provide valuable information of simplified algorithm for an influential biomarker panel.

\section{Methods}

A retrospective protocol of objects, literature research strategies, inclusion and exclusion criteria, outcome measurements, and methods of statistical analysis was conducted adhering to the previous recommended guidelines and was written in accordance with the Preferred Reporting Items for Systematic Reviews and Meta-analysis (PRISMA) checklist. ${ }^{16}$

\section{Literature search strategy}

Literature research was conducted in March 2017 for articles published from 2002 to 2017 without restriction to regions, language, and publication type. Electronic databases of PubMed, Embase, Web of Science, ScienceDirect, Springer, Wiley, ProQuest, and Ovid were searched by two authors independently. Detailed PubMed strategy was listed as follows: risk factor, renal toxicity/nephrotoxicity, calcineurin inhibitors/tacrolimus/cyclosporine, and transplant. Related articles of the included studies were screened to broaden the research with Google Scholar applied for the identification of risk factors. Moreover, computer research was supplemented with manual researches for all retrieved studies, reviews, and conference abstracts. When multiple reports studying the same group of participants were picked up, the latest one was enrolled.

\section{Inclusion and exclusion criteria}

To ensure the homogeneity across studies, the involved studies had to meet specific criteria. All eligible retrospective studies (cohort/case-control) should indicate CNI nephrotoxicity after renal transplantation, which are based on the double or triple immunosuppression therapy consisting of CNI (FK506/CsA) and antiproliferative agent (mycophenolate mofetil $[\mathrm{MMF}]$ /azathioprine $[\mathrm{AZA}]$ ) with/without steroids (methylprednisolone [MP]/prednisone [Pred]). The CNI nephrotoxicity was diagnosed with explicit protocol biopsy or through medical record. Potential risk factors of CNI nephrotoxicity were defined with clear odds ratio 
(OR) and 95\% CI through multivariate logistic analysis (univariate, if not available). The editorials, reviews, animal experiments, conference abstracts, and pediatric publications were excluded, and the randomized controlled trials (RCTs), although having lower confounding effects than the nonrandomized studies (observational), were dismissed for limited number and potential hybrid effects. ${ }^{15,17}$

\section{Data extraction and outcome of interest}

Data from involved studies were analyzed by two independent authors (Xia and Zhu). For any discrepancy, another author (Zhang) was resorted to for adjudication. The following primary outcomes were comprehensively identified as CNI nephrotoxicity: ${ }^{15,18,19}$ 1) a persistent elevated Scr of at least $1.6 \mathrm{mg} / \mathrm{dL}$ and/or a creeping Scr level of $\sim 20 \%$ from the baselines indicating renal dysfunction, with a subsequent decrease after conversion to an immunosuppressive-free protocol; 2) biopsy-proven subclinical nephropathy and progression of chronicity score according to the Banff criteria; 3) exclusion of result from AR either proven by biopsy or judged by clinical evaluation; and 4) acute deterioration of renal perfusion diagnosed by Doppler ultrasound of the graft. The secondary outcome was enrolled as delayed graft function (DGF), which was reckoned as a perioperative combination of immunosuppression regime. DGF was defined as the need for dialysis within the first week after transplantation and/ or a creatinine clearance of $<10 \mathrm{~mL} / \mathrm{min}$ during the first postoperative 3 days.

\section{Quality assessment and statistical analysis}

Quality assessment was carried out for all retrieved studies. Quality in the systematic review referred to the potential of biases during data analysis. The methodological integrity of the study was carried out according to the "NewcastleOttawa Scale (NOS) for assessing the quality of nonrandomized studies in meta-analysis," ${ }^{20}$ which included three main criteria, namely patient selection, comparability of the studied group, and assessment of the enrolled trials. A score ranging from 0 to 9 (presented as stars) was allocated for each retrospective observational study, and the studies that obtain more than six stars were reckoned to be of high quality.

Meta-analysis was performed with Review Manager (version 5.2; Nordic Cochrane Centre, The Cochrane Collaboration, Copenhagen, Denmark). Heterogeneity was assessed using the Cochrane $\mathrm{Q}$ and $I^{2}$ statistics. $p>0.10$ using the $\chi^{2}$ test for the Q statistic and $I^{2}<50 \%$ for the $I^{2}$ statistic were interpreted as low-level heterogeneity. A pooled effect was calculated with a fixed-effects model when there was no statistically significant heterogeneity; otherwise, a randomeffects model was employed. ${ }^{21}$

Subgroup analysis was achieved for comparison of the results from different regions. Sensitivity analysis was conducted on the weight allocated for different CNI nephrotoxicity endpoints for donor age, and the funnel plots were obtained to screen for potential publication bias.

\section{Results}

In general, 12 trials with 2,849 cases (911 with CNI nephrotoxicity and 1,892 without CNI nephrotoxicity, data from one trial not available) fulfilled the predefined criteria and were included in the final analysis (Figure 1). ${ }^{18,19,22-31}$ Full text of the 12 studies was retrieved for further extraction, and the titles and abstracts were inspected.

\section{Characteristics of eligible studies}

Basic information of the eligible studies was given in Table 1. Among these studies, there were two retrospective case-control studies and 10 cohort studies. CNI nephrotoxicity was biopsy defined in five studies, index decided (eGFR/Scr/FK506 concentration) in four studies, and DGF-designated in three studies. The enrolled centers were mainly oriented in Asian and European countries. All the participants received a CNI-based double or triple therapy after medication.

\section{Methodological quality of eligible studies}

Quality of the enrolled studies was generally high with an NOS score $>6$. All clarified the length of patient follow-up, and CNI nephrotoxicity was explicitly diagnosed. Despite this, few matching information was obtained in most studies. Four studies included relative small sample size $(n<100)$, and the results of handling missing data for some were not adequately discussed. NOS evaluation for each study from three perspectives was presented in Table 2.

\section{Outcome measurements}

First, we conducted a meta-analysis of 12 retrospective analyses regarding the risk factor with posttransplantation CNI nephrotoxicity versus the nontoxicity group. The pooled data offered insight to 15 risk factors associated with posttransplantation CNI toxicity including donor age, donor gender (male), recipient age, recipient body mass index (BMI), cold ischemia time (CIT), human leukocyte antigen (HLA) mismatch, hypertension, diabetes mellitus, zero-time arteriosclerosis, infectious disease, $\mathrm{AR}$, recipient $C Y P 3 A 5 * 3 / * 3$ genotype, $A B C B 11236 C>T, A B C B 13435 C>T$, and donor 
ScienceDirect: 1,324; Web of Science: 406; ProQuest: 899;

PubMed: 222; Embase: 432; Springer: 612; Wiley: 23; Ovid: 614

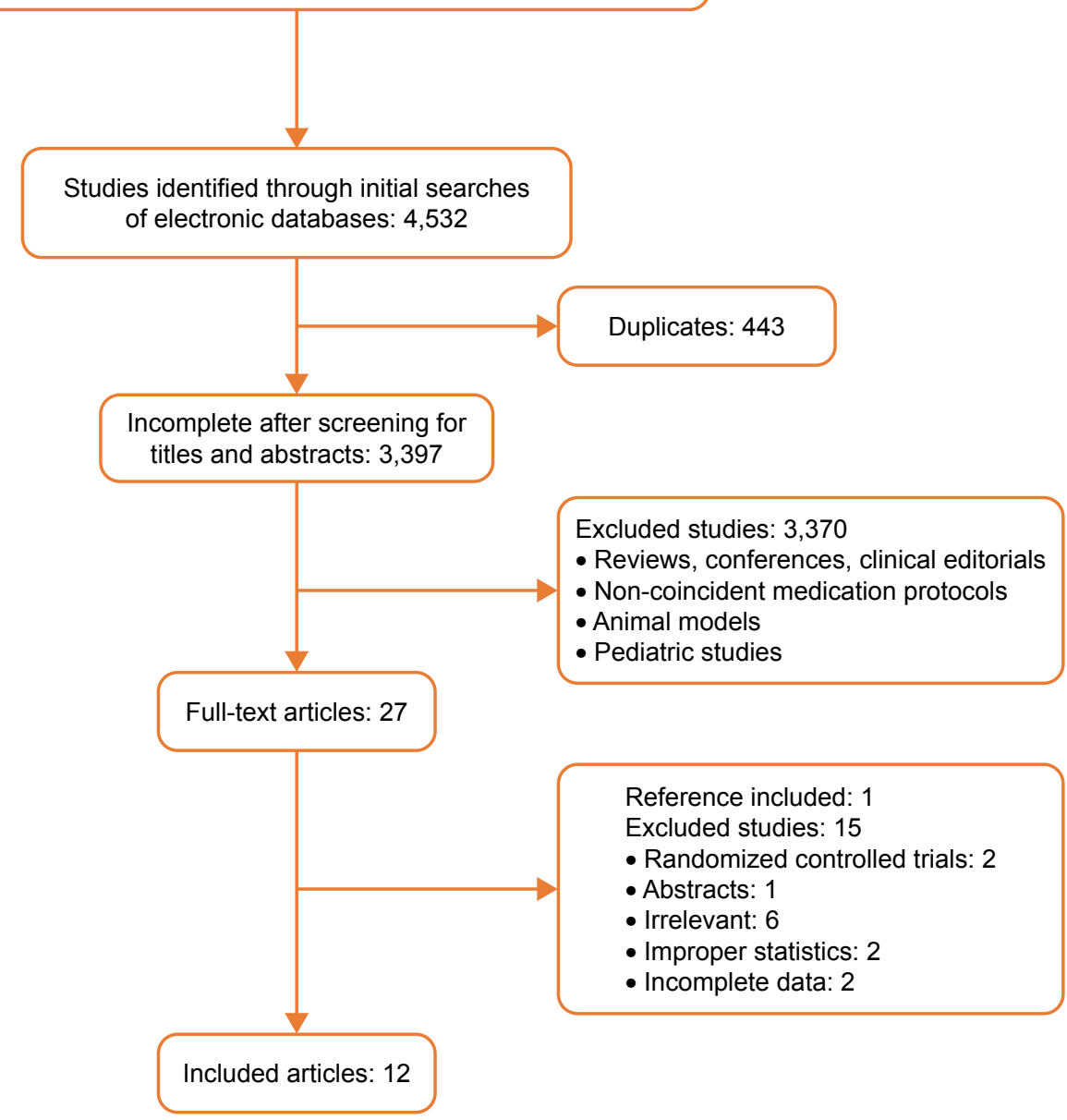

Figure I Flow diagram of studies identified, included, and excluded.

$A B C B 13435 C>T$ allele mutants (Table 3), with the classification given in Table 4.

Forest plot of the pooled results demonstrated three significant risk factors for CNI nephrotoxicity, namely donor age (OR, 1.01; 95\% CI, 1.01-1.03; $p=0.02$ ), recipient zerotime arteriosclerosis (OR, 1.44; 95\% CI, 1.04-1.99; $p=0.03)$, and $C Y P 3 A 5 * 3 / * 3$ genotype (OR, 2.80; 95\% CI, 2.63-2.98; $p=0.00$; Figure 2). Donor age was mentioned by six cohort studies in European and Asian countries as a risk factor with statistical significance. Fixed-effects model was adopted considering the little heterogeneity $\left(\chi^{2}=1.74 ; p=0.88 ; I^{2}=0 \%\right)$ between studies. Biopsy-proven zero-time arteriosclerosis exhibited a tight correlation with CNI nephrotoxicity through fixed-effects model analysis $\left(\chi^{2}=0.00 ; p=0.96 ; I^{2}=0 \%\right)$. Both the donor and recipient genetic polymorphisms were investigated in this analysis, and only recipient $C Y P 3 A 5 * 3 / * 3$ genotype $\left(\chi^{2}=0.05 ; p=0.83 ; I^{2}=0 \%\right)$ might bring about a high risk of posttransplantation CNI nephrotoxicity.

\section{Subgroup analysis}

Subgroup analysis was carried out for comparison of original results with donor age as a risk factor in European and Asian populations (Figure 3). Interestingly, donor age exhibited correlation with CNI nephrotoxicity in both European (OR, 1.02; 95\% CI, 1.00-1.03) and Asian (OR, 1.01; 95\% CI, 1.00-1.03) countries, in addition to its significance as a risk factor for all-case incidence. The other two risk factors (recipient zero-time arteriosclerosis and $C Y P 3 A 5 * 3 / * 3$ genotype) were only reported by two articles, making it difficult to perform subgroup analysis.

In addition, identification of donor age as a risk factor with the primary and secondary outcomes was inspected in subgroup analysis (recipient zero-time arteriosclerosis and CYP3A5 nonexpressor were both identified according to the primary outcome). It was noteworthy that donor age turned to be an influential risk factor for CNI nephrotoxicity with the primary outcome $(\mathrm{OR}, 1.01 ; 95 \% \mathrm{CI}, 1.00-1.03)$ rather 


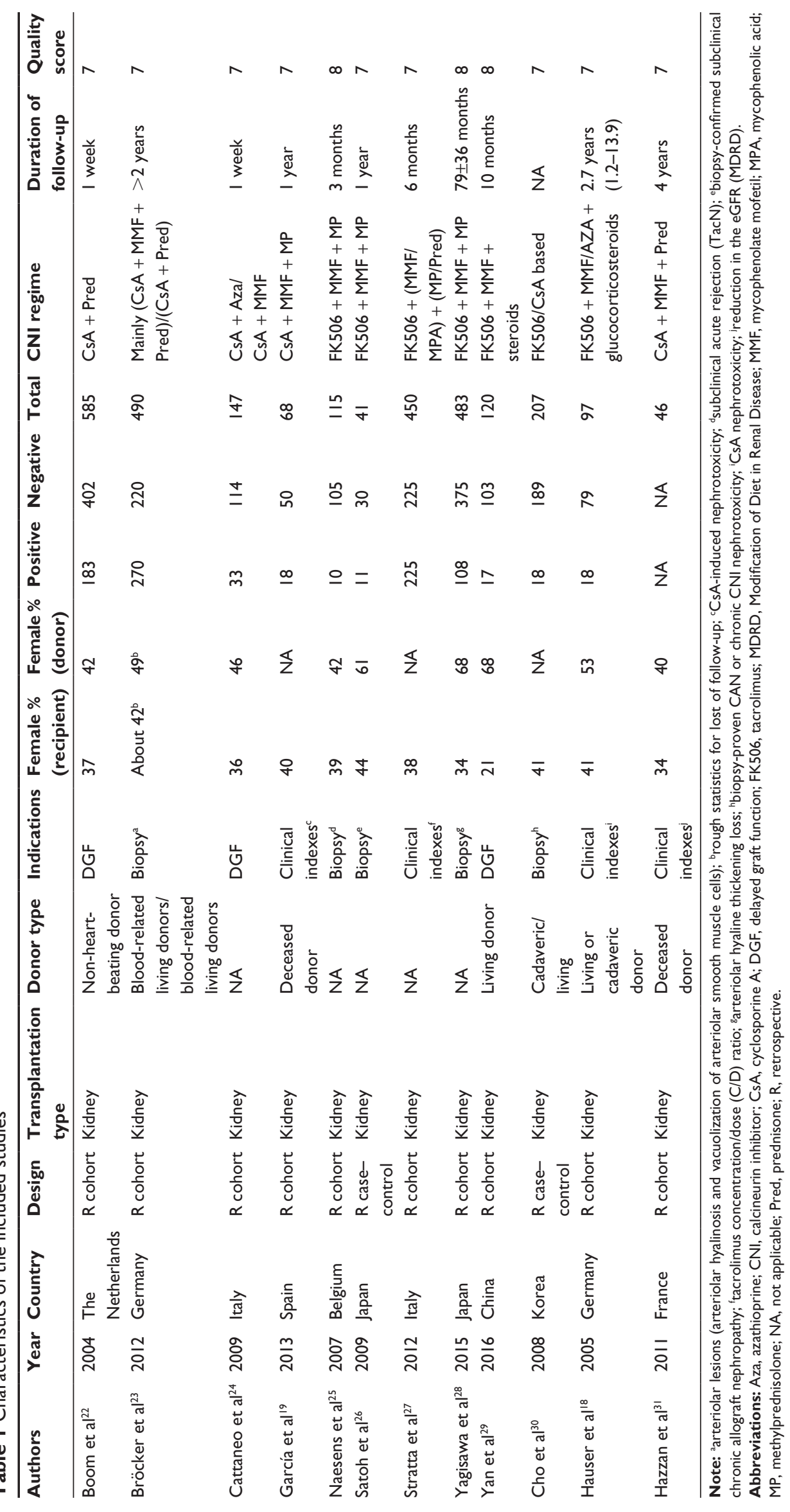




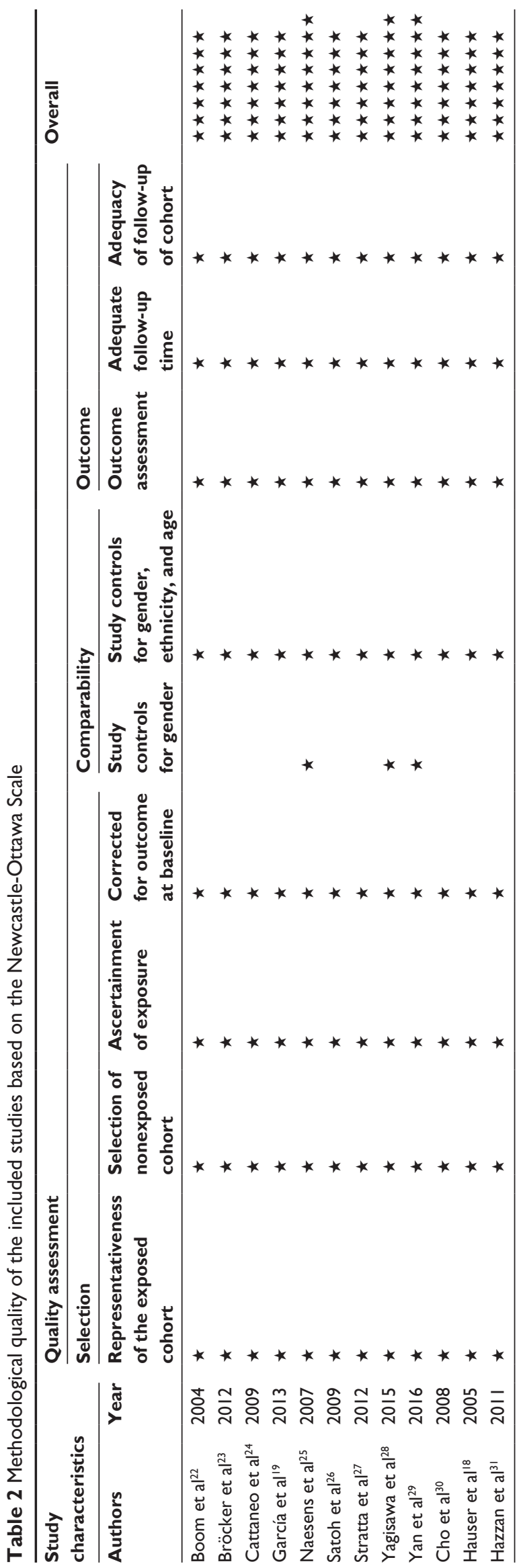

than the secondary outcome (OR, 1.02; 95\% CI, 0.99-1.05; Figure 4).

\section{Sensitivity analysis and publication bias}

The enrolled retrospective studies that scored $>6$ on the NOS checklist were included for sensitivity analysis. Herein, sensitivity analysis excluding each individual study at a time obtained similar results (data not shown), indicating that the sensitivity was low and identification of donor age as a risk factor was reliable.

Figure 5 presented the funnel plot of studies proposing donor age as a risk factor for CNI nephrotoxicity. All studies lie inside the $95 \% \mathrm{CI}$, with an even distribution around the vertical in the upper part, indicating no obvious publication bias.

\section{Discussion}

This meta-analysis of 12 retrospective studies containing 2,894 adult patients highlighted three risk factors in correlation with CNI nephrotoxicity after solid organ transplantation. Older donor age, recipient zero-time arteriosclerosis, and $C Y P 3 A 5 * 3 / * 3$ genotype might probably expose the patients to a higher risk for CNI nephrotoxicity.

Among the confirmed risk factors in this review, donor age seemed to be of particular relevance with clinical outcomes. Grafts from older donors might exhibit age-reduced functional capability and an increased sensitivity toward CNI regime, ${ }^{32}$ which might contribute to the nonimmunologic $\mathrm{CNI}$ nephrotoxicity after transplantation and reduce long-term graft outcome. As stated by Naesens et $a 1,{ }^{33}$ donor age might exert even more influential effect than the quality of the graft at implantation and continue to be important for histologic and functional evolution in posttransplantation kidney decay. The impact of donor age could be explained by several physiological and pathological factors. Primarily, aging implied a reduction of nephron number in accompany with a reduced kidney reserve. ${ }^{34}$ The unavoidable kidney erosion with time was doomed with its ill function. In spite of the apparent normal structure at transplantation, the renal cells from older kidney might reach their cycling limit in short term and experience specific molecular and morphologic changes with the negative effect from progressive failure of cellular repair mechanism. ${ }^{35}$ Second, increased susceptibility of older donor kidneys was observed for CNI nephrotoxicity with several coinciding phenomena. ${ }^{36}$ The progress in chronic pathology with age could be attributed to a higher susceptibility not only to typical transplantation-related injury such as ischemia and reperfusion but also to downstream ischemic phenomena 
Table 3 Results of pooled outcomes

\begin{tabular}{|c|c|c|c|c|c|c|c|}
\hline \multirow[t]{2}{*}{ Outcomes of interest } & \multirow{2}{*}{$\begin{array}{l}\text { Number } \\
\text { of studies }\end{array}$} & \multicolumn{2}{|l|}{ Mean difference } & \multicolumn{4}{|c|}{ Study heterogeneity } \\
\hline & & Mean $(95 \% \mathrm{Cl})$ & $p$-value & $x^{2}$ & $d f$ & $I^{2}(\%)$ & $p$-value \\
\hline Donor age & 6 & $1.01(1.00-1.03)$ & 0.02 & 1.74 & 5 & 0 & 0.88 \\
\hline Donor gender (male) & 3 & $1.19(0.86-1.65)$ & 0.28 & 0.28 & 2 & 0 & 0.87 \\
\hline Recipient age & 3 & $1.01(0.98-1.04)$ & 0.47 & 0.44 & 2 & 0 & 0.80 \\
\hline Recipient BMI & 2 & $1.18(0.96-1.45)$ & 0.12 & 0.02 & 1 & 0 & 0.90 \\
\hline Cold ischemia time & 3 & $1.00(1.00-1.00)$ & 0.37 & 1.42 & 2 & 0 & 0.49 \\
\hline HLA mismatch & 3 & $1.04(0.79-1.38)$ & 0.76 & 1.69 & 4 & 0 & 0.79 \\
\hline Hypertension & 2 & $0.93(0.67-1.31)$ & 0.69 & 1.12 & 2 & 0 & 0.57 \\
\hline Diabetes mellitus & 2 & $0.93(0.5 \mathrm{I}-1.69)$ & 0.81 & 0.59 & 1 & 0 & 0.44 \\
\hline Arteriosclerosis & 3 & $1.44(1.04-1.99)$ & 0.03 & 1 & 1 & 0 & 0.96 \\
\hline Infectious disease & 2 & $1.22(0.76-1.96)$ & 0.41 & 0.84 & 2 & 0 & 0.66 \\
\hline Acute rejection & 2 & $1.27(0.83-1.96)$ & 0.27 & 0 & 1 & 0 & 0.97 \\
\hline Recipient CYP3A5*3/*3 & 3 & $2.79(2.62-2.97)$ & 0 & 1.96 & 2 & 0 & 0.38 \\
\hline Recipient $A B C B|| 236 C>T$ & 2 & $1.12(0.59-2.11)$ & 0.74 & 0.1 & 1 & 0 & 0.75 \\
\hline Recipient $A B C B / 3435 C>T$ & 2 & $1.39(0.76-2.54)$ & 0.29 & 0.33 & 1 & 0 & 0.56 \\
\hline Donor $A B C B / 3435 C>T$ & 2 & $0.73(0.29-1.84)$ & 0.50 & 1.72 & 1 & 42 & 0.19 \\
\hline
\end{tabular}

Abbreviations: BMI, body mass index; HLA, human leukocyte antigen.

and further amplification of the effect from donor age on the histologic evolution of transplanted kidneys. Furthermore, the vasodilator and vasoconstrictory responses triggered by CNI were amplified in an older kidney when CNI metabolic clearance was reduced with aging. ${ }^{33,37}$ It should be noted that older donors had become an essential part of the donor pool, especially in the "extended criteria of donors" era. The older donor might provide an alternative for the ESRD patients when kidney source was in short. Therefore, inclusion of clinical determinants of the donor age would improve predictive accuracy for CNI nephrotoxicity to relieve patients from severe renal injury. Specifically, despite donor age was reckoned to be a risk factor for CNI nephrotoxicity (OR, $1.01 ; 95 \%$ CI, $1.01-1.03 ; p=0.02$ ), further subgroup analysis claimed its significance only with the primary outcome (OR, $1.01 ; 95 \%$ CI, 1.00-1.03) rather than the secondary outcome (OR, 1.02; 95\% CI, 0.99-1.05). It was then hypothesized that CNI-induced DGF was a complication subject to donor sources and operational circumstances (machine perfusion, CIT, etc.) more than donor age. ${ }^{38,39}$

In this study, recipient zero-time arteriosclerosis was proposed as a risk factor together with donor age. It was consistent with the hypothesis that arteriolar lesions in early transplant biopsy samples with zero-time arteriosclerosis

Table 4 Classification for the pooled outcomes of potential risk factors

\begin{tabular}{|c|c|c|c|c|c|c|c|c|c|}
\hline \multirow[t]{2}{*}{ Outcomes of interest } & \multicolumn{2}{|c|}{ Demographic } & \multicolumn{2}{|c|}{ Genetic } & \multicolumn{3}{|c|}{$\begin{array}{l}\text { Environmental } \\
\text { (operation) }\end{array}$} & \multirow[t]{2}{*}{ Immune } & \multirow[t]{2}{*}{ Nonimmune } \\
\hline & Donor & Recipient & CYP & ABCB I & Pre & Peri & Post & & \\
\hline Donor age & $\sqrt{ }$ & & & & $\sqrt{ }$ & & & & $\sqrt{ }$ \\
\hline Donor gender (male) & $\sqrt{ }$ & & & & $\sqrt{ }$ & & & & $\sqrt{ }$ \\
\hline Recipient age & & $\sqrt{ }$ & & & $\sqrt{ }$ & & & & $\sqrt{ }$ \\
\hline Recipient BMI & & $\sqrt{ }$ & & & $\sqrt{ }$ & & & & $\sqrt{ }$ \\
\hline Cold ischemia time & & $\sqrt{ }$ & & & & $\sqrt{ }$ & & & $\sqrt{ }$ \\
\hline HLA mismatch & & $\sqrt{ }$ & & & & $\sqrt{ }$ & & $\sqrt{ }$ & \\
\hline Hypertension & & $\sqrt{ }$ & & & $\sqrt{ }$ & & & & $\sqrt{ }$ \\
\hline Diabetes mellitus & & $\sqrt{ }$ & & & $\sqrt{ }$ & & & & $\sqrt{ }$ \\
\hline Arteriosclerosis & & $\sqrt{ }$ & & & $\sqrt{ }$ & & & & $\sqrt{ }$ \\
\hline Infectious disease & & $\sqrt{ }$ & & & $\sqrt{ }$ & & & & $\sqrt{ }$ \\
\hline Acute rejection & & $\sqrt{ }$ & & & & $\sqrt{ }$ & & $\sqrt{ }$ & \\
\hline Recipient CYP3A5*3/*3 & & $\sqrt{ }$ & $\sqrt{ }$ & & $\sqrt{ }$ & & & & $\sqrt{ }$ \\
\hline Recipient $A B C B / \quad \mid 236 C>T$ & & $\sqrt{ }$ & & $\sqrt{ }$ & $\sqrt{ }$ & & & & $\sqrt{ }$ \\
\hline Recipient $A B C B / 3435 C>T$ & & $\sqrt{ }$ & & $\sqrt{ }$ & $\sqrt{ }$ & & & & $\sqrt{ }$ \\
\hline Donor $A B C B I 3435 C>T$ & $\sqrt{ }$ & & & $\sqrt{ }$ & $\sqrt{ }$ & & & & $\sqrt{ }$ \\
\hline
\end{tabular}

Abbreviations: BMI, body mass index; HLA, human leukocyte antigen. 


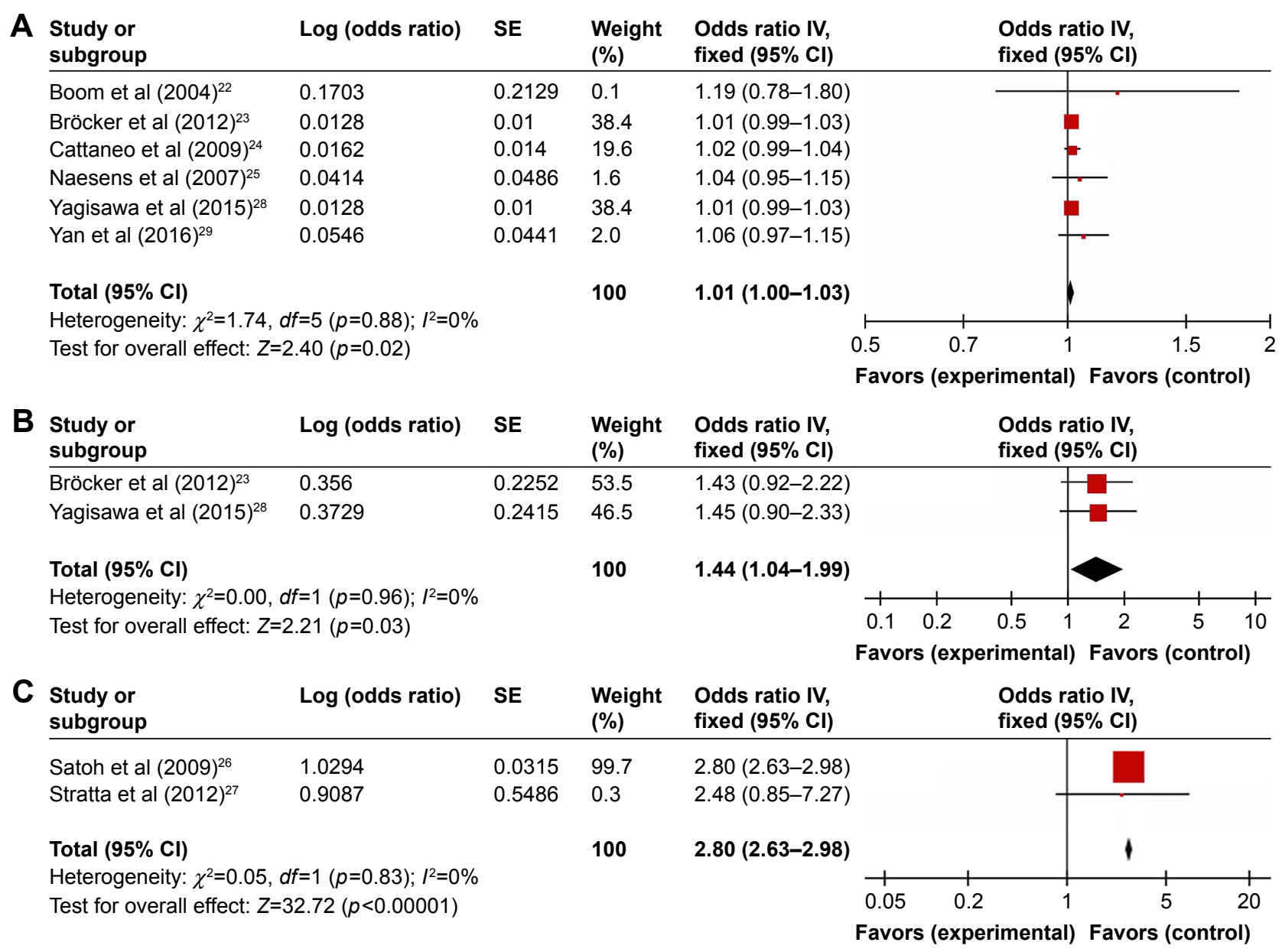

Figure 2 Forest plot and meta-analysis of risk factors for CNI nephrotoxicity.

Note: $(\mathbf{A})$ donor age, $(\mathbf{B})$ recipient zero-time arteriosclerosis, and $(\mathbf{C})$ recipient $C Y P 3 A 5 * 3 / * 3$ genotype.

Abbreviations: CNI, calcineurin inhibitor; IV, inverse variance.

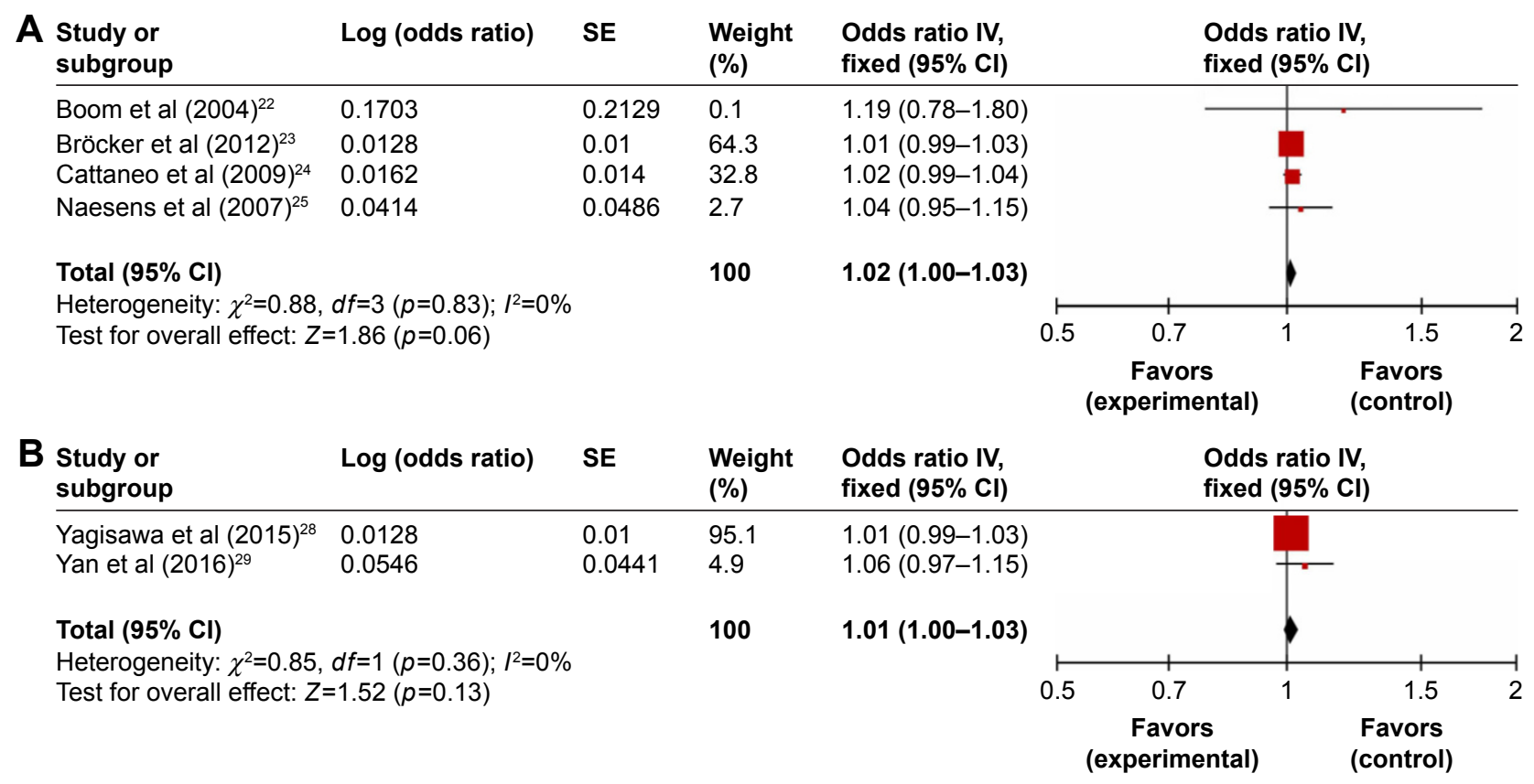

Figure 3 Subgroup analysis of donor age as a risk factor for CNI nephrotoxicity in Caucasian and Asian populations.

Note: (A) Caucasian and (B) Asian.

Abbreviations: $\mathrm{CNI}$, calcineurin inhibitor; IV, inverse variance. 


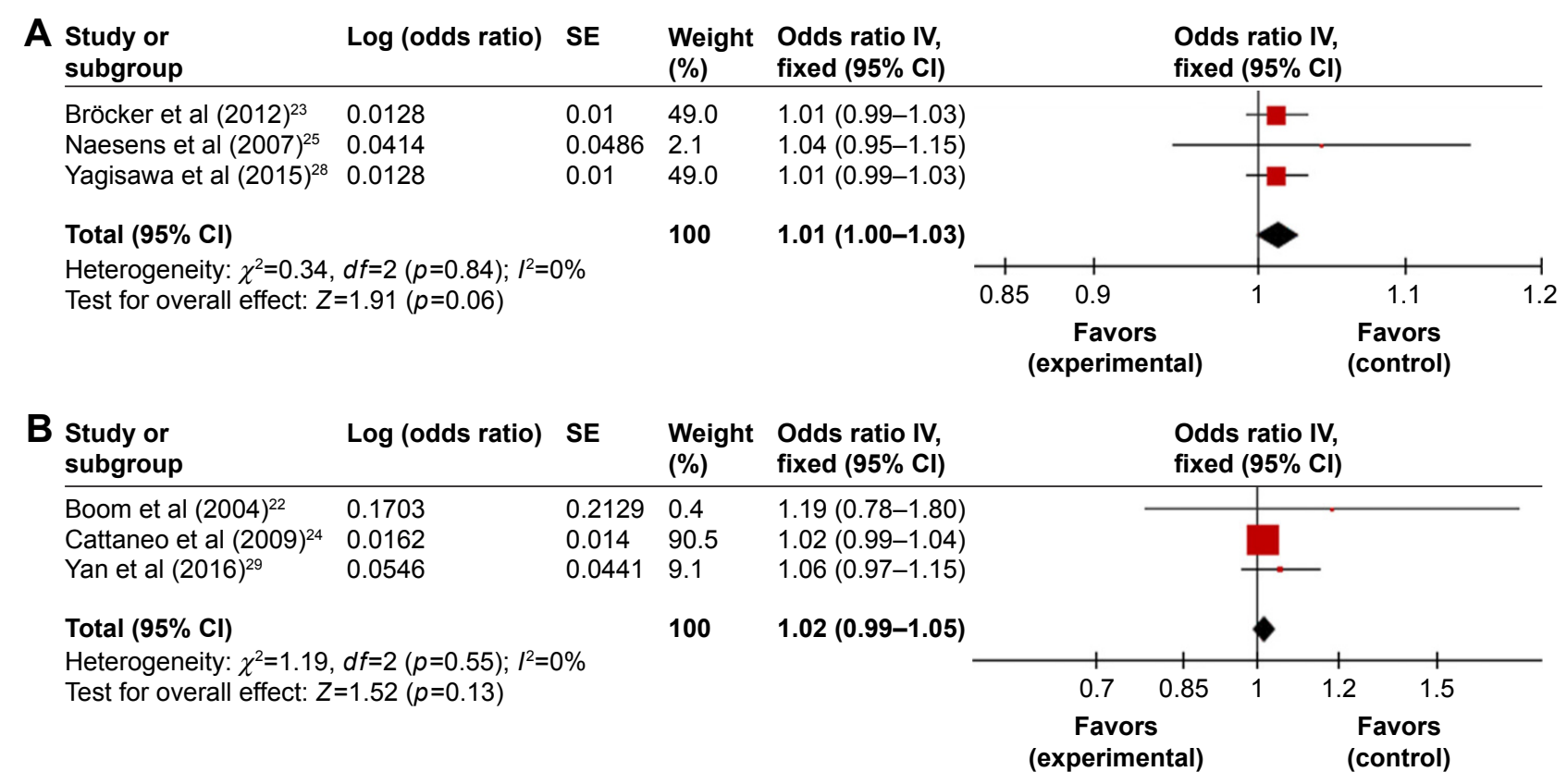

Figure 4 Subgroup analysis of donor age as a risk factor for CNI nephrotoxicity with the primary and secondary outcome.

Note: (A) Primary and (B) secondary.

Abbreviation: $\mathrm{CNI}$, calcineurin inhibitor.

were age-related or due to long-standing arterial hypertension, presumably in the donor. ${ }^{23}$ Shimizu and coworkers affirmed the preexistence of moderate- to severe-grade interlobular zero-time arteriosclerosis in an allograft as a risk factor for the development of acute FK506-induced nephrotoxicity. ${ }^{28}$ Zero-time arteriosclerosis, identified by biopsy as fibrous intimal thickening of arcuate and interlobular arteries, was associated with transforming growth factor- $\beta$ (TGF- $\beta$ ) and other profibrogenic molecules, ${ }^{40}$ and the fibrotic processes were exactly reckoned as the hallmarks of CNIinduced long-term nephrotoxicity. What was more, TGF- $\beta$ was closely associated with angiotensin II, which might lead to an uncontrolled activation of the renin-angiotensin

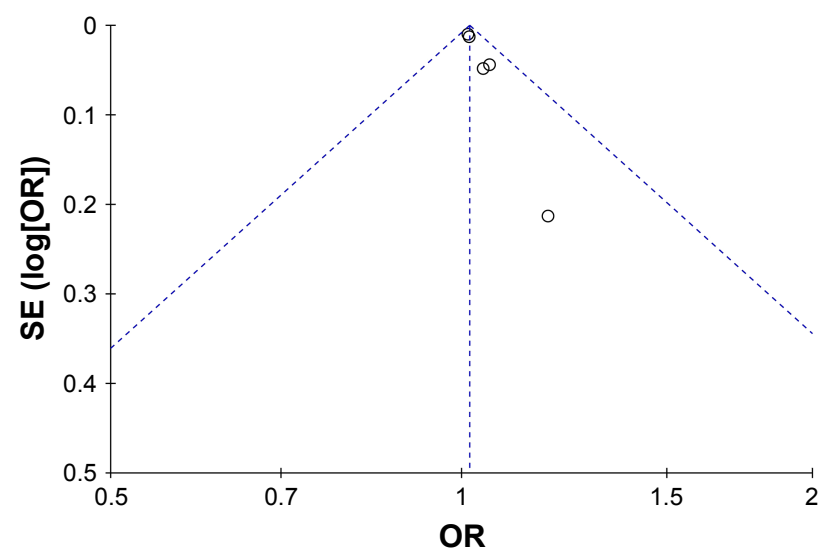

Figure 5 Funnel plot illustrating donor age as a risk factor for $\mathrm{CNI}$ nephrotoxicity. Abbreviations: $\mathrm{CNI}$, calcineurin inhibitor; $\mathrm{OR}$, odds ratio. system (RAS). The imbalance between vasoconstrictors and vasodilators could ultimately lead to hemodynamic changes and eventually irreversible structural damage..$^{41}$ Although it had been challenged that the histologic lesions from CNIs exerted little prognostic value on long-term kidney outcome and the absence of CNI lesions might be associated with nonadherence or even inferior long-term outcomes, ${ }^{42,43}$ zero-time arteriosclerosis at transplantation might expose the posttransplant allograft to an ill-functioning microenvironment.

Recipient $C Y P 3 A 5 * 3 / * 3$ genotype was summarized as another risk factor for $\mathrm{CNI}$ nephrotoxicity in recessive model analysis, coinciding with the pharmacogenetic basis of $\mathrm{CNI}$ metabolism. CNIs underwent extensive CYP3A5 metabolism in liver and small intestine (a little in kidney), expression and activity of which were only meaningful in patients possessing at least one $C Y P 3 A 5^{*} 1$ allele $\left(C Y P 3 A 5^{*} 1 / * 1\right.$ or $C Y P 3 A 5^{*} 1 / * 3$ genotype, defined as $C Y P 3 A 5$ expressor), compared with the CYP $3 A 5 * 3$ mutant patients (CYP $3 A 5 * 3 / * 3$ genotype, defined as $C Y P 3 A 5$ nonexpressor) ${ }^{44}$ Therefore, altered bioavailability and system clearance of CNI were identified in CYP3A5 expressors with an average $30 \%$ increased oral clearance resulting in $50 \%$ higher dose requirement, ${ }^{45}$ posing the nonexpressors to a higher risk of CNI nephrotoxicity with elevated CNI system exposure.

In clinical practice of most centers, therapeutic drug monitoring (TDM) was universally applied to guide CNI medication, to reach a predefined target concentration with optimal balance between efficacy and toxicity. ${ }^{46}$ However, it was 
worth mentioning that the relationship of dose exposurenephrotoxicity in CNI regime was more complicated than it appeared to be, making TDM a less influential indicator of CNI nephrotoxicity. On the one hand, the nonlinear relationship between dosage and exposure brought about a large interindividual variability in CNI PK. A combination of demographic, clinical, genetic, and drug-drug interaction information through a predictive algorithm would allow for more reliable CNI dosing. ${ }^{47}$ On the other hand, even in the predefined therapeutic window, the predictive value of TDM results for the occurrence of CNI nephrotoxicity was limited. The "jet lag" of TDM with a period of supra/under-dosage especially in the perioperative phase might be responsible for CNI-induced SAR and DGF. ${ }^{45}$ In this review, recipient $C Y P 3 A 5 * 3 / * 3$ genotype with higher CNI system exposure was annotated as a risk factor for CNI nephrotoxicity. Although the exposure-nephrotoxicity relationship was not elucidated and whether system or local exposure of CNI was responsible for CNI still remain controversial, the results in this study indicated that $C Y P 3 A 5$ genotype could influence the PD characters of FK506 metabolism, which lay beyond an efficient control of clinical TDM. Furthermore, evidence suggested that local FK506 exposure in the kidney, instead of the system exposure, should be probably incriminated for the nephrotoxic effects.

Of note, several prospects must be taken into account. Primarily, current researches posed a main focus on the recipients, whereas graft quality and donor genetic polymorphism might not be negligible for kidney outcome. Although donor profiles might provide a direct reflection on CNI local exposure, the relative high heterogeneity of limited studies hitherto needed further validation. Second, CNIs were substrates of CYP3A and P-gp (ATP-binding cassette subfamily B member 1, ABCB1), for both the enzymes share broad specificities. ${ }^{48}$ In this study, nonsupportive results were observed of any $\mathrm{ABCB} 1$ mutants $(1236 \mathrm{C}>\mathrm{T}$, $2677 \mathrm{G}>\mathrm{A} / \mathrm{T}$, and $3435 \mathrm{C}>\mathrm{T}$ ) as risk factors for CNI nephrotoxicity in Caucasian populations. This could be explained that MDR1 polymorphisms in normal volunteers exhibited significant differences in the genotype or allele frequencies among different ethnic groups. C1236T was located in exon 12 within ABCB1 with $\mathrm{CC}$ genotype more frequent than TT genotype in Caucasians, while less common in Asians. $A B C B 12677 G>T / A$ was also one of the most common and extensively reported single-nucleotide polymorphisms (SNPs). Previous research had shown that the A allele was observed in $13 \%$ of Chinese, $2 \%$ of Caucasians, and $0 \%$ of Africans. ${ }^{30}$ Moreover, allele frequencies of SNP exon $263435 \mathrm{C}>\mathrm{T}$ among different populations ranged from a
$66 \% \mathrm{~T}$ allele frequency in South-west Asians (Indian) to 16\% $\mathrm{T}$ allele frequency in African Americans, followed by a decline in East Asians (Chinese and Malays). ${ }^{49}$ It could be then concluded that the potential influence of ABCB1 genotype was to be uncovered in Asian populations. Also, from a statistic point of view, the more variables were included, and the larger sample size was required for biomarker validation. Invalid or poorly explanatory variables might in turn reduce the robustness of the integrated biomarker system. Therefore, this innovative study provided convincing clues for biomarker identification of CNI nephrotoxicity.

Undoubtedly, there were several limitations in this metaanalysis. The main limitation was that all enrolled studies were retrospective, for which inadequate random sequence generation and binding might introduce the risk of clinical bias. More systematic reviews were expected in the future when enough RCTs were available. Moreover, patents with different definitions of CNI nephrotoxicity including the biopsy-based ones, index-based ones, and DGF were included without stratification and assessed together. The noninterchangable diagnostic tools of the enrolled observational studies might lead to confusing interpretation, and other complications or combined drug use in the clinical setting could compromise result validity. Specifically, the secondary outcome DGF had always been a controversial topic. ${ }^{17}$ The enrolled studies in this meta-analysis claimed CNI nephrotoxicity to blame for DGF, and the ELITESymphony Study conducted by Ekberg et al ${ }^{50}$ propose delayed allograft function as CNI efficacy endpoints although DGF was not necessarily CNI dose dependent. Herein, the primary and secondary outcomes were discriminated in subgroup analysis, and the results indicated donor age as a risk factor only in protocol biopsy or index-based clinical diagnosis rather than DGF. Also, other potential risk factors identified from both primary and secondary definitions failed to get through further analysis due to limited papers $(n=2$, one from primary outcome and the other from secondary outcome for recipient age, BMI, etc.). However, although the abovementioned drawbacks might affect the interpretability of the meta-analysis, the strictly conducted strategies to identify studies and the predefined criteria for methodological control proved fit for the purpose of identifying risk factors for CNI nephrotoxicity and provided preliminary clues for CNI nephrotoxicity in the posttransplant complications after initiation of a CNI-based immunotherapy.

\section{Conclusion}

Overall, CNIs remain the backbone of current immunosuppression regimes despite the association with nephrotoxicity, 
and CNI nephrotoxicity could be alerted through a robust integrated biomarker system for earlier, preemptive treatment. This meta-analysis outlined three independent risk factors for the development of CNI nephrotoxicity in organ transplantation patients, including older donor age, recipient zero-time arteriosclerosis, and $C Y P 3 A 5 * 3 / * 3$ genotype. A thorough examination of these three risk factors was beneficial to detect early aberration of pathological alterations and facilitate with preemptive clinical intervention in addition to TDM. Nevertheless, despite the rigorous methodology, the inherent limitations of the retrospective observational studies and incoherent definitions of CNI nephrotoxicity of the limited trials enrolled held us back from more convincing conclusions. Large-size and well-designed RCTs with welldefined CNI nephrotoxicity endpoints and extensive followups were in urgent need for a comprehensive exploration of pretransplantation, peritransplantation, and posttransplantation risk factors for timely clinical intervention.

\section{Acknowledgments}

This work was financed by the National Natural Science Foundation of China (81573793), Program of International S\&T Cooperation (2015DFA31810), Shanghai Shenkang Hospital Development Center Project (SHDC12015120), Beijing Medical Award Foundation (YJHYXKYJJ-122), and Shanghai Key Specialty Project of Clinical Pharmacy (2016-40044-002). We were also grateful to Jimmy Chan and Wan Xiao (Agilent, Shanghai) for their incessant support.

\section{Author contributions}

Feng Zhang and Wansheng Chen conceived the study. Shouhong Gao, Mingming Li, and Xia Tao collaborated on data collection and data analysis. Sang Zhu conducted data analysis. Tianyi Xia drafted this article. All authors approved the final version of the submitted manuscript. All authors contributed toward data analysis, drafting and critically revising the paper and agree to be accountable for all aspects of the work.

\section{Disclosure}

The authors report no conflicts of interest in this work.

\section{References}

1. Vincenti F. Reduced exposure to calcineurin inhibitors in renal transplantation. N Engl J Med. 2007;357(25):2562.

2. Nankivell BJ, Kuypers DR. Diagnosis and prevention of chronic kidney allograft loss. Lancet. 2011;378(9800):1428.

3. Zununi Vahed S, Ardalan M, Samadi N, Omidi Y. Pharmacogenetics and drug-induced nephrotoxicity in renal transplant recipients. Bioimpacts. $2015 ; 5(1): 45-54$.
4. Joy MS, Hogan SL, Thompson BD, Finn WF, Nickeleit V. Cytochrome P4503A5 expression in the kidneys of patients with calcineurin inhibitor nephrotoxicity. Nephrol Dial Transplant. 2007;22(7):1963.

5. Lusco MA, Fogo AB, Najafian B, Alpers CE. AJKD atlas of renal pathology: calcineurin inhibitor nephrotoxicity. Am J Kidney Dis. 2017; 69(5):e21.

6. Vázquez LC, González AP, Juega J, et al. Nodular arteriolar hyalinosis as histopathologic hallmark of calcineurin inhibitor nephrotoxicity: does it always have the same meaning? Transplant Proc. 2015;47(8):2357-2360.

7. Kashani K, Al-Khafaji A, Ardiles T, et al. Discovery and validation of cell cycle arrest biomarkers in human acute kidney injury. Crit Care. 2013;17(1):R25.

8. Aregger F, Uehlinger DE, Witowski J, et al. Identification of IGFBP-7 by urinary proteomics as a novel prognostic marker in early acute kidney injury. Kidney Int. 2014;85(4):909-919.

9. Halloran PF, Famulski KS, Reeve J. Molecular assessment of disease states in kidney transplant biopsy samples. Nat Rev Nephrol. 2016;12(9): 534-548.

10. Roodnat JI, Hilbrands LB, Smak Gregoor PJ, et al. 15-year follow-up of a multicenter, randomized, calcineurin inhibitor withdrawal study in kidney transplantation. Transplantation. 2014;98(1):47-53.

11. Merville P. Combating chronic renal allograft dysfunction. Drugs. 2005;65(5):615-631.

12. Vliet JAVD, Warlé MC, Cheung CLS, Teerenstra S, Hoitsma AJ. Influence of prolonged cold ischemia in renal transplantation. Clin Transplant. 2011;25(6):E612.

13. Yates PJ, Nicholson ML. The aetiology and pathogenesis of chronic allograft nephropathy. Transpl Immunol. 2006;16(3):148-157.

14. Glowacki F, Lionet A, Hammelin JP, et al. Influence of cytochrome P450 3A5 (CYP3A5) genetic polymorphism on the pharmacokinetics of the prolonged-release, once-daily formulation of tacrolimus in stable renal transplant recipients. Clin Pharmacokinet. 2011;50(7):451.

15. Jacobson PA, Schladt D, Israni A, et al. Genetic and clinical determinants of early, acute calcineurin inhibitor-related nephrotoxicity: results from a kidney transplant consortium. Transplantation. 2012;93(6):624.

16. Liberati A, Altman DG, Tetzlaff J, et al. The PRISMA statement for reporting systematic reviews and meta-analyses of studies that evaluate healthcare interventions: explanation and elaboration. BMJ. 2009; 6(4):e1-e34.

17. Elens L, Bouamar R, Hesselink DA, Haufroid V, van Gelder T, van Schaik RH. The new CYP3A4 intron $6 \mathrm{C}>\mathrm{T}$ polymorphism (CYP3A4*22) is associated with an increased risk of delayed graft function and worse renal function in cyclosporine-treated kidney transplant patients. Pharmacogenet Genomics. 2012;22(5):373-380.

18. Hauser IA, Schaeffeler E, Gauer S, et al. ABCB1 genotype of the donor but not of the recipient is a major risk factor for cyclosporine-related nephrotoxicity after renal transplantation. J Am Soc Nephrol. 2005; 16(5):1501.

19. García M, Macías RM, Cubero JJ, Benítez J, Caravaca F, Gervasini G. $\mathrm{ABCB} 1$ polymorphisms are associated with cyclosporine-induced nephrotoxicity and gingival hyperplasia in renal transplant recipients. Eur J Clin Pharmacol. 2013;69(3):385.

20. Taggart DP, D'Amico R, Altman DG. Effect of arterial revascularisation on survival: a systematic review of studies comparing bilateral and single internal mammary arteries. Lancet. 2001;358(9285):870-875.

21. Higgins JP, Green S. Cochrane handbook for systematic reviews of interventions. Naunyn-Schmiedebergs Archiv für experimentelle Pathologie und Pharmakologie. 2008;5(2):S38.

22. Boom H, Mallat MJ, de Fijter JW, Paul LC, Bruijn JA, van Es LA. Calcium levels as a risk factor for delayed graft function. Transplantation. 2004;77(6):868.

23. Bröcker V, Schubert V, Scheffner I, et al. Arteriolar lesions in renal transplant biopsies: prevalence, progression, and clinical significance. Am J Pathol. 2012;180(5):1852-1862.

24. Cattaneo D, Ruggenenti P, Baldelli S, et al. ABCB1 genotypes predict cyclosporine-related adverse events and kidney allograft outcome. J Am Soc Nephrol. 2009;20(6):1404-1415. 
25. Naesens M, Lerut E, Damme BV, Vanrenterghem Y, Kuypers DRJ. Tacrolimus exposure and evolution of renal allograft histology in the first year after transplantation. Am J Transplant. 2007;7(9):2114-2123.

26. Satoh $\mathrm{S}$, Saito M, Inoue $\mathrm{T}$, et al. CYP3A5 *1 allele associated with tacrolimus trough concentrations but not subclinical acute rejection or chronic allograft nephropathy in Japanese renal transplant recipients. Eur J Clin Pharmacol. 2009;65(5):473.

27. Stratta P, Quaglia M, Cena T, et al. The interactions of age, sex, body mass index, genetics, and steroid weight-based doses on tacrolimus dosing requirement after adult kidney transplantation. European Journal of Clinical Pharmacology, 2012;68(5):671-680.

28. Yagisawa T, Omoto K, Shimizu T, Ishida H, Tanabe K. Arteriosclerosis in zero-time biopsy is a risk factor for tacrolimus-induced chronic nephrotoxicity. Nephrology. 2015;20(Suppl 2):51.

29. Yan L, Li Y, Tang JT, An YF, Wang LL, Shi YY. Donor ABCB1 3435 $\mathrm{C}>\mathrm{T}$ genetic polymorphisms influence early renal function in kidney transplant recipients treated with tacrolimus. Pharmacogenomics. 2016;17(3):249-257.

30. Cho JH, Huh S, Kwon TG, Choi JY. Association of C-509T and T869C polymorphisms of transforming growth factor- $\beta 1$ gene with chronic allograft nephropathy and graft survival in Korean renal transplant recipients. Transplant Proc. 2008;40(7):2355.

31. Hazzan M, Hertig A, Buob D, et al. Epithelial-to-mesenchymal transition predicts cyclosporine nephrotoxicity in renal transplant recipients. J Am Soc Nephrol. 2011;22(7):1375.

32. De-Fijter J, Mallat M, Doxiadis I, et al. Increased immunogenicity and cause of graft loss of old donor kidneys. J Am Soc Nephrol. 2001; 12(7):1538.

33. Naesens M, Lerut E, De JH, Van DB, Vanrenterghem Y, Kuypers DR. Donor age and renal P-glycoprotein expression associate with chronic histological damage in renal allografts. J Am Soc Nephrol. 2009;20(11): 2468.

34. Nyengaard JR, Bendtsen TF. Glomerular number and size in relation to age, kidney weight, and body surface in normal man. Anat Rec. 1992; 232(2):194.

35. Jennings $\mathrm{P}$, Koppelstaetter C, Aydin S, et al. Cyclosporine A induces senescence in renal tubular epithelial cells. Am J Physiol. 2007; 293(3):F831-F838

36. Moreso F, Serón D, Gilvernet S, et al. Donor age and delayed graft function as predictors of renal allograft survival in rejection-free patients. Nephrol Dial Transplant. 1999;14(4):930.

37. Burdmann EA, Andoh TF, Yu L, Bennett WM. Cyclosporine nephrotoxicity. Kidney Int. 2003;23(5):465-476.
38. Matos ACC, Borrelli M, Nogueira M, et al. Impact of machine perfusion after long static cold storage on delayed graft function incidence and duration and time to hospital discharge. Clin Translant. Epub 2017 Oct 3.

39. Lim WH, Mcdonald SP, Russ GR, et al. Association between delayed graft function and graft loss in donation after cardiac death kidney transplants-a paired kidney registry analysis. Transplantation. 2017; 101(6):1139-1143.

40. Goldfarb DA. Expression of TGF-beta and fibrogenic genes in transplant recipients with tacrolimus and cyclosporine nephrotoxicity. Kidney Int. 2003;169(6):2436.

41. Fernando M, Peake PW, Endre ZH. Biomarkers of calcineurin inhibitor nephrotoxicity in transplantation. Biomark Med. 2014;8(10):1247.

42. Einecke G, Reeve J, Halloran PF. Hyalinosis lesions in renal transplant biopsies: time-dependent complexity of interpretation. Am J Transplant. 2016;17(5):1346-1357.

43. Mengel M, Mihatsch M, Halloran PF. Histological characteristics of calcineurin inhibitor toxicity - there is no such thing as specificity! Am J Transplant. 2011;11(12):2549-2550.

44. Haufroid V, Wallemacq P, Vankerckhove V, et al. CYP3A5 and ABCB1 polymorphisms and tacrolimus pharmacokinetics in renal transplant candidates: guidelines from an experimental study. Am J Transplant. 2006;6(11):2706-2713.

45. Hesselink DA, Van GT, van Schaik RH. The pharmacogenetics of calcineurin inhibitors: one step closer toward individualized immunosuppression? Pharmacogenomics. 2005;6(4):323-337.

46. Hesselink DA, Bouamar R, Elens L, van Schaik RH, Van GT. The role of pharmacogenetics in the disposition of and response to tacrolimus in solid organ transplantation. Clin Pharmacokinet. 2014;53(2):123.

47. Birdwell KA, Decker B, Barbarino JM, et al. Clinical pharmacogenetics implementation consortium (CPIC) guidelines for CYP3A5 genotype and tacrolimus dosing. Clin Pharmacol Ther. 2015;98(1):19-24.

48. Englund G, Lundquist P, Skogastierna C, et al. Cytochrome p450 inhibitory properties of common efflux transporter inhibitors. Drug Metab Dispos. 2014;42(3):441.

49. Tang K, Ngoi SM, Gwee PC, et al. Distinct haplotype profiles and strong linkage disequilibrium at the MDR1 multidrug transporter gene locus in three ethnic Asian populations. Pharmacogenetics. 2002; 12(6):437-450.

50. Ekberg H, Tedesco-Silva H, Demirbas A, et al; ELITE-Symphony Study. Reduced exposure to calcineurin inhibitors in renal transplantation. N Engl J Med. 2007;357(25):2562.
Drug Design, Development and Therapy

\section{Publish your work in this journal}

Drug Design, Development and Therapy is an international, peerreviewed open-access journal that spans the spectrum of drug design and development through to clinical applications. Clinical outcomes, patient safety, and programs for the development and effective, safe, and sustained use of medicines are the features of the journal, which

\section{Dovepress}

has also been accepted for indexing on PubMed Central. The manuscript management system is completely online and includes a very quick and fair peer-review system, which is all easy to use. Visit http://www.dovepress.com/testimonials.php to read real quotes from published authors. 